\title{
Propriedades não destrutivas do MDP produzido com partículas de madeiras e de llex paraguariensis
}

\author{
Non-destructive properties of MDP produced \\ with wood particles and llex paraguariensis
}

\author{
Joel Telles de Souza ${ }^{1}$, Maiara Talgatti ${ }^{2}$, Walmir Marques de Menezes ${ }^{2}$, \\ Clóvis Roberto Haselein ${ }^{3}$, Elio José Santini ${ }^{3}$ e Rafael Beltrame ${ }^{4}$
}

\begin{abstract}
Resumo
A diversificação das bases para a fabricação de painéis, por meio de novos produtos a partir da utilização de resíduos, poderá beneficiar o setor florestal, ao passo que a fabricação industrial poderá aumentar, sem que haja necessariamente a implantação de novos maciços florestais. Sendo assim, o presente estudo tem como objetivo avaliar a viabilidade da utilização de resíduos madeireiros da industrialização da erva-mate (Ilex paraguariensis a. st. hil.), para a manufatura de painéis MDP, em combinação com diferentes proporções de madeira de Eucalyptus grandis e Pinus elliottii, coladas com ureia-formaldeído. Para realização desse trabalho, foram utilizados resíduos madeireiros de erva-mate, oriundas de Barão de Cotegipe - RS e partículas de madeira de pinus e eucalipto, oriundas do abate de árvores de plantios florestais da Universidade Federal de Santa Maria- UFSM. Todo o processo de produção dos painéis MDP no laboratório de produtos florestais da UFSM, os testes não destrutivos de ultrassom e colorimetria foram realizados na Universidade Federal de Pelotas. O método de avaliação não destrutiva utilizando ultrassom pode ser utilizado na predição das propriedades físicas dos painéis MDP e para as propriedades mecânicas de flexão estática. Por fim, a colorimetria apresentou coloração rosada para painéis de eucalipto e erva-mate, e coloração amarelada para os produzidos com pinus e erva-mate. Assim, conclui-se que, o resíduo madeireiro de erva-mate em combinação com as espécies comumente utilizadas em painéis comerciais, pinus e eucalipto, pode ser utilizado em painéis MDP sem prejudicar a qualidade dos mesmos, além disso, a mistura não interfere de maneira pronunciada na coloração dos painéis obtidos.
\end{abstract}

Palavras-chave: resíduos, painéis aglomerados, colorimetria, ultrassom.

\begin{abstract}
The diversification of the foundations for the manufacture of panels, through new products from the use of waste, could benefit the forestry sector, while industrial manufacturing could increase, without necessarily implementing new forest stands. The present study aims to evaluate the feasibility of the use of wood residues from the industrialization of yerba mate (Ilex paraguariensis a. st. hil.), for the manufacture of MDP panels, in combination with different proportions of wood of Eucalyptus grandis and Pinus elliottii, bonded with urea-formaldehyde. In order to carry out this work, wood residues of yerba mate, from Barão de Cotegipe - RS and pine and eucalyptus wood particles, were obtained from forest plantations at the Federal University of Santa Maria. The entire process of production of the MDP panels took place at the UFSM forest products laboratory, the non destructive ultrasound and colorimetry tests were performed at the Federal University of Pelotas. The non-destructive evaluation method using ultrasound can be used to predict the physical properties of MDP panels and the mechanical properties of static bending. Finally, the colorimetry presented a pink coloration for eucalyptus panels and a yellowish coloration for those produced with pine and yerba mate. Therefore, it is concluded that the woody residue of yerba mate in combination with the species commonly used in commercial panels, pine and eucalyptus, can be used in MDP panels without harming the quality of the same.
\end{abstract}

Keywords: waste, agglomerated panels, colorimetry, ultrasoun.

${ }^{1}$ Professor Adjunto do Departamento de Ciências Florestais. UNOESC - Universidade do Oeste de Santa Catarina. Rua Getúlio Vargas, 2125 - Flor da Serra, 89600-000 - Joaçaba, SC, Brasil.

${ }^{2}$ Doutorando (a) em Engenharia Florestal. UFSM - Universidade Federal de Santa Maria / Centro de Ciências Rurais. Av. Roraima, 1000 - Camobi - 97105-900 - Santa Maria, RS, Brasil. E-mail: maiara.talgatti@hotmail.com; menzeswalmis@hotmail.com.

3Professor Titular do Departamento de Ciências Florestais. UFSM - Universidade Federal de Santa Maria / Centro de Ciências Rurais. Av. Roraima, 1000 - Camobi - 97105-900 - Santa Maria, RS, Brasil. E-mail: haselein@ hotmail.com; santini@hotmail.com

${ }^{4}$ Professor Adjunto do Departamento de Ciências Florestais. UFPel - Universidade Federal de Pelotas / Centro de Engenharias. Rua Conde de Porto Alegre, 793 - 96010-290 - Pelotas, RS, Brasil. E-mail: beltramerafael@ hotmail.com.

Sci. For., Piracicaba, v. 46, n. 119, p. 507-518, set. 2018 DOI: dx.doi.org/10.18671/scifor.v46n119.17 


\section{INTRODUÇÃO}

$\mathrm{Na}$ indústria de produtos florestais o uso de tecnologias não destrutivas de materiais, podem auxiliar na implementação de programas de classificação de compostos de madeira, resultando em um material engenheirado, com desempenho e características bem definidas (HAN et al., 2006). A previsibilidade quanto à performance estrutural dessa classe de produtos é indispensável para o tipo de uso que lhe é atribuído e pode ser atingida utilizando diferentes técnicas já consolidadas de avaliações não destrutivas, as quais possibilitam estimar as propriedades dos materiais e auxiliam nas definições de seu uso adequado (ROSS et al., 2004).

Os métodos de avaliação não destrutivos podem ser definidos como aqueles que identificam as propriedades dos materiais sem alterar suas características estruturais e, consequentemente, sua capacidade de uso final. Como vantagens proporcionadas por esses métodos podem ser citados: a estimativa das propriedades sem a destruição de peças; a obtenção de informações que possam ser utilizadas para tomada de decisão no uso adequado de peças estruturais; adequabilidade para uso em linhas de produção; rapidez e praticidade na obtenção dos resultados (PELLERIN; ROSS, 2002). Tais aspectos fazem das técnicas não destrutivas importantes ferramentas na caracterização da madeira e compostos de madeira, podendo ser empregados pelas indústrias para melhorar o controle de qualidade dos produtos por meio de uma maior uniformidade na matéria-prima e em seus derivados.

Conforme descrito por Oliveira et al. (2003), os avanços tecnológicos têm permitido melhorar o aproveitamento da madeira e de seus derivados, criando novos produtos e ampliando sua aplicação. Dentre esses avanços, pode-se destacar a técnica de ultrassom como uma importante ferramenta para classificar peças estruturais de madeira, e dessa forma, permitir um incremento na qualidade e na competitividade desse material. No entanto, para isso se faz necessária a compreensão do fenômeno de propagação das ondas ultra-sônicas no material, mediante estabelecimento de relações entre as diversas variáveis envolvidas.

Acrescenta-se ainda, que a percepção da qualidade de um produto está relacionada com a sua utilização. Um dos principais critérios de determinação da qualidade é a aparência superficial, principalmente se o uso final da madeira for a fabricação de móveis, de assoalhos ou de objetos decorativos. Padrões específicos de cores e desenhos são apontados como tendências e modismos na indústria moveleira, tornando o visual do material um fator limitante na escolha de uma espécie. Para a determinação da cor o método quantitativo mais utilizado é a colorimetria, a ciência da medição da cor (GONÇALEZ et al., 2001).

Em vista do exposto acima, este capítulo tem por objetivo, estimar as propriedades físico-mecânicas por meio do ultrassom e determinar a variação da cor por meio de colorímetro, em painéis MDP (Medium Density Particleboard) produzidos com diferentes proporções de resíduos madeireiros de erva-mate (Ilex paraguariensis), em combinação com Eucalyptus grandis e Pinus elliottii.

\section{MATERIAL E MÉTODOS}

\section{Coleta e preparo da matéria-prima}

Para realização deste trabalho, foram utilizadas partículas de madeira de Ilex paraguariensis A. St. Hil., Eucalyptus grandis W. Hill ex Maiden, Pinus elliottii Engelm e adesivo à base de ureia-formaldeído.

O resíduo madeireiro de erva-mate foi cedido pela empresa Ervateira Barão de Cotegipe Ltda., da cidade de Barão de Cotegipe-RS. Esse material foi transportado para o Laboratório de Produtos Florestais (LPF) da Universidade Federal de Santa Maria (UFSM), onde foi processado em moinho de martelos equipado de peneira com orifícios de $8,0 \mathrm{~mm}$ de diâmetro, para as partículas de camada interna (Figura $1 \mathrm{C}$ ); e peneira de 1,0 mm de diâmetro, para as partículas de camada externa (Figura 1B). Por fim, o material foi submetido à peneiragem com abertura de malha de 0,150 mm, para descarte das partículas mais finas (Figura 1 A). As partículas utilizadas foram as que não passaram pela malha da peneira. 


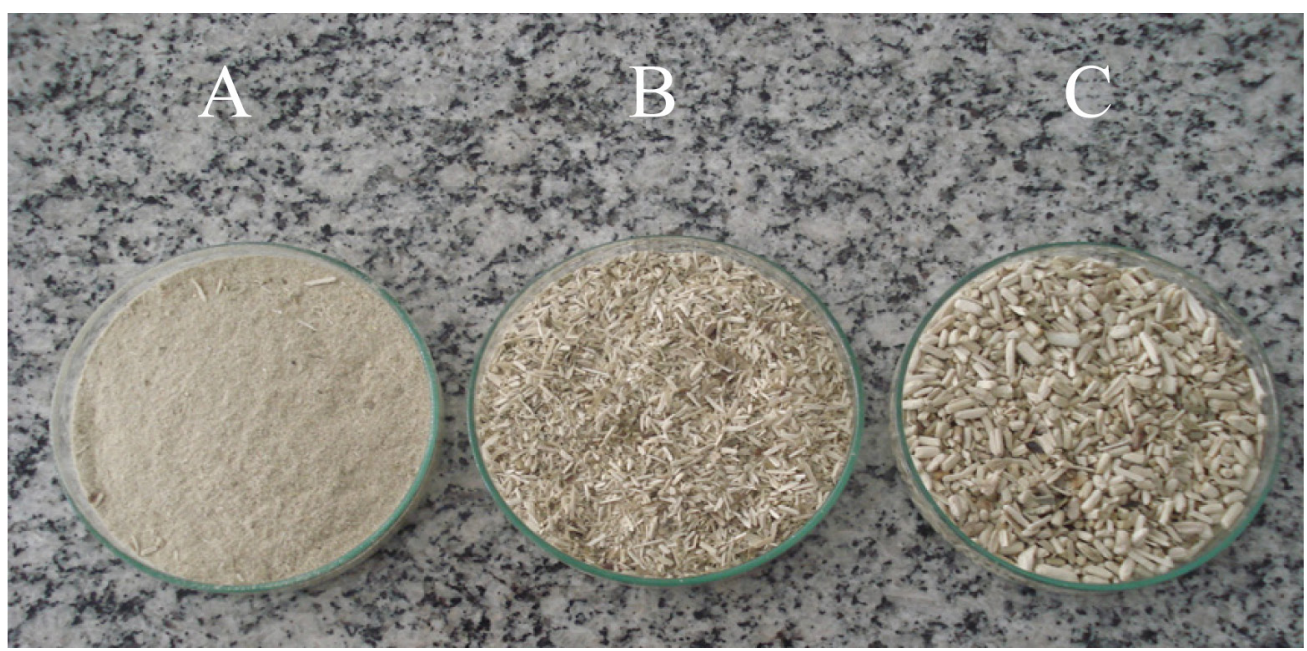

Figura 1. Resíduo madeireiro de erva-mate após o processamento e peneiragem. Descarte das partículas mais finas - pó (A); partículas da camada externa (B) e partículas da camada interna (C).

Figure 1. Yerba mate residue after processing and sieving. Discarding the finer particles - powder $(A)$; particles of the outer layer (B) and particles of the inner layer (C).

As espécies Eucalyptus grandis W. Hill ex Maiden e Pinus elliottii Engelm foram obtidas em povoamentos florestais homogêneos de aproximadamente 15 anos, localizados no Campus da UFSM. Foram selecionadas três árvores de cada espécie, de fuste reto e boas condições fitossanitárias. Após o abate, foi retirado de cada árvore as duas primeiras toras com comprimento de 2,70 m cada. Posteriormente, procedeu-se ao desdobro das toras com a retirada de tabuas de $5 \mathrm{~cm}$ de espessura, as quais foram resserradas para obtenção de blocos com $5 \mathrm{~cm}$ de espessura, $7 \mathrm{~cm}$ de comprimento e largura variável de acordo com o diâmetro e a posição de obtenção das tábuas na tora.

Esses blocos de madeira foram imersos em água por dez dias, com o objetivo de saturar a madeira, facilitando assim o corte e a obtenção de lascas inteiras. A obtenção das lascas foi realizada em flaker (moinho de facas), com corte ajustado para 0,55 mm de espessura, obtendo-se dimensões finais das lascas de aproximadamente 50 x 70 x 0,55 mm. Após, esse material foi submetido à secagem ao ar por 15 dias, sendo posteriormente reduzido a partículas em moinho de martelos, equipado de peneira com orifícios de $8,0 \mathrm{~mm}$ de diâmetro, para as partículas de camada interna (Figura 2C); e peneira de 1,0 mm de diâmetro, para as partículas de camada externa (Figura 2B). Por fim, o material foi peneirado com abertura de malha de 0,150 $\mathrm{mm}$, para descarte das partículas mais finas (Figura 2A). As partículas utilizadas foram as que não passarem pela malha da peneira.

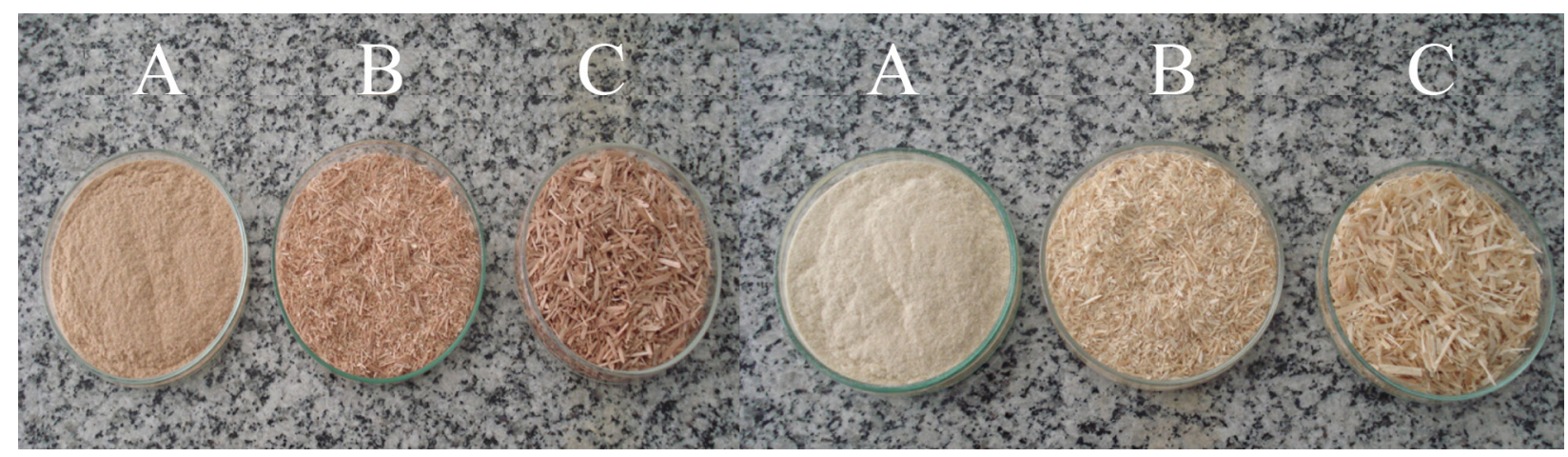

Figura 2. Partículas de Eucalyptus grandis (à esquerda em coloração rosada) e Pinus elliottii (à direita em coloração amarelada), após o processamento e peneiragem. Descarte das partículas mais finas - pó (A); partículas da camada externa (B) e partículas da camada interna (C).

Figure 2. Particles of Eucalyptus grandis (left in pink) and Pinus elliottii (right in yellowish color), after processing and sieving. Discarding the finer particles - powder $(A)$; particles of the outer layer $(B)$ and particles of the inner layer $(C)$.

Foi utilizado adesivo à base de ureia-formaldeído. Tal adesivo, foi industrialmente produzido com um teor de sólidos de aproximadamente 65\%. 


\section{Processo de produção dos painéis MDP}

Após a preparação das partículas, as mesmas foram condicionadas em estufa a $60^{\circ} \mathrm{C}$ durante pelo menos 24 horas, até alcançar a umidade de equilíbrio de aproximadamente $3 \%$. Como se trata de painel MDP em camadas, a composição do mesmo foi pré-estabelecida da seguinte maneira:

- Camada externa (CE): $86 \%$ de partículas, $13 \%$ de adesivo e $1 \%$ de parafina;

- Camada interna (CI): $91 \%$ de partículas, $8 \%$ de adesivo e $1 \%$ de parafina.

Para ambas as camadas, trabalhou-se com uma massa específica de 0,65 g.cm ${ }^{-3}$ a um teor de umidade de equilíbrio de $8 \%$.

As partículas de cada camada foram imediatamente levadas a um tambor rotativo e misturadas nas diferentes proporções. O adesivo e a parafina na forma líquida foram aplicados por meio de pistola acionada por compressor de ar, com pressão de 8 kgf.cm ${ }^{-2}$ e vazão de 50 g.minuto ${ }^{-1}$. Juntamente com o adesivo e a parafina, foi aplicada a água necessária para ajustar o teor de umidade do colchão a aproximadamente $12 \%$ para camada externa e $8 \%$, para camada interna. Gerada a mistura que dava origem ao colchão, a mesma foi pesada e levada a um molde de madeira de $50 \times 50 \times 20 \mathrm{~cm}$, onde foi realizada uma pré-prensagem manual a temperatura ambiente por aproximadamente 2 minutos.

O colchão formado foi então para a prensagem definitiva, realizada em prensa hidráulica. Foi

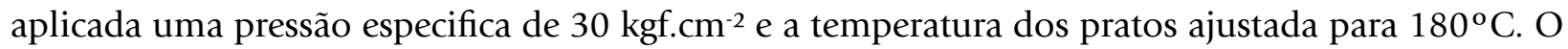
tempo de fechamento da prensa foi de 40 segundos e o tempo total de prensagem aplicado para promover a evaporação da água e a cura do adesivo foi de 12 minutos.

A espessura do painel foi estabelecida por duas barras de aço com altura de $1,50 \mathrm{~cm}$, as quais foram colocadas nas laterais do colchão, limitando o fechamento da prensa. Após a prensagem, os painéis foram dispostos em câmara climatizada $\left(20^{\circ} \mathrm{C}\right.$ e $65 \%$ de umidade relativa) até atingir peso constante.

\section{Ensaio não destrutivo com ultrassom}

Foram realizados os ensaios não destrutivos com um equipamento portátil de ultrassom, dotado de transdutores de pontos secos com frequência de aproximadamente $50 \mathrm{kHz}$, que medem diretamente o tempo de propagação das ondas, em microssegundos $(\mu \mathrm{s})$. A determinação do tempo de propagação das ondas foi realizada pelo método de transmissão direta.

As leituras do tempo de propagação da onda, foram obtidas a partir da razão entre a média das leituras e o comprimento do trecho percorrido pela onda; assim, foi calculada a velocidade de propagação, em metros por segundo $(\mathrm{m} / \mathrm{s})$. O cálculo do módulo de elasticidade dinâmico (MOED) pelo método de propagação direto, foi por meio da Equação 1. O MOED foi correlacionado com as características físicas e mecânicas dos corpos de prova.

$$
M O E D=\frac{V P^{2} \times D A}{G} \times 10^{-5}
$$

Em que: $\mathrm{MOED}=$ Módulo de elasticidade dinâmico (MPA); VP = Velocidade de propagação da onda $\left(\mathrm{m} \cdot \mathrm{s}^{-1}\right) ; \mathrm{DA}=$ Densidade aparente $\left(\mathrm{kg} \cdot \mathrm{m}^{-3}\right) ; g=$ aceleração da gravidade, $9,804 \mathrm{~m} \cdot \mathrm{s}^{-2}$.

\section{Ensaio de colorimetria}

A avaliação colorimétrica das amostras ocorreu por meio de colorímetro digital pertencente à Universidade Federal de Pelotas. O aparelho foi configurado para o uso de fonte de luz D65 e ângulo de observação de $10^{\circ}$ no sistema CIELAB 1976. Foram realizadas seis medições em cada corpo de prova, sendo que para cada tratamento houve quatro repetições, perfazendo assim, um total de 24 medições por tratamento.

O colorímetro fornece, em seu display, as seguintes variáveis: "L*", correspondente ao grau de claridade ( $0=$ preto até $100=$ branco); $\mathrm{a}^{*}$ e b*, correspondentes as coordenadas verde-vermelho ("-a" $\left.e^{\prime \prime}+a^{\prime \prime}\right)$ e azul-amarelo ("-b" $\left.e^{\prime \prime}+b^{\prime \prime}\right)$, respectivamente. A partir das variáveis $L^{*}, a^{*}$ e b*, calculou-se os valores C (saturação da cor) e $\mathrm{H}^{*}$ (ângulo de tinta ou tonalidade, o qual define a cor final do corpo de prova).

\section{Delineamento experimental}

Objetivando avaliar o efeito das partículas madeireiras de erva-mate na manufatura de painéis MDP, foi combinando 11 diferentes proporções de partículas de Ilex paraguariensis, com Eucalyptus grandis e Pinus elliottii, coladas com ureia-formaldeído. Foram avaliados 11 tratamentos (Tabela 1), 
com quatro repetições cada, perfazendo um total de 44 painéis MDP, com as dimensões de 50 x 50 $\mathrm{x} 1,50 \mathrm{~cm}$, todos confeccionados com massa especifica nominal de $0,65 \mathrm{~g} . \mathrm{cm}^{-3}$.

Tabela 1. Tratamentos utilizados no experimento.

Table 1. Treatments used in the experiment.

\begin{tabular}{lccc}
\hline Tratamentos & & Partículas (\%) & Eucalipto \\
\cline { 2 - 4 } & Erva-mate & Pinus & 0 \\
T1 & 0 & 100 & 0 \\
T2 & 25 & 75 & 0 \\
T3 & 50 & 50 & 0 \\
T4 & 75 & 25 & 0 \\
T5 & 100 & 0 & 100 \\
T6 & 0 & 0 & 75 \\
T7 & 25 & 0 & 50 \\
T8 & 50 & 0 & 25 \\
T9 & 75 & 0 & 33,3 \\
T10 & 33,3 & 33,3 & 50 \\
T11 & 0 & 50 & \\
\hline
\end{tabular}

\section{Análise estatística dos dados}

Os resultados dos ensaios foram submetidos à análise de correlação simples de Pearson a 5\% de probabilidade de erro. Após, buscou-se o ajuste de equações lineares que visavam estimar o desempenho dos painéis MDP quanto a sua resistência superficial observadas nos ensaios destrutivos, em função do MOED obtido por meio do ultrassom.

Para avaliar o efeito dos diferentes tratamentos sobre os parâmetros colorimétricos dos painéis MDP, fez-se uma análise de variância e, quando significativa, foi aplicado o teste de média de Scott - Knott, a 5\% de probabilidade de erro. Optou-se pelo teste de Scott - Knott para melhor interpretação dos resultados, já que o presente experimento possui um grande número de tratamentos, havendo interesse numa separação real de grupos de médias, sem a ambiguidade de resultados.

\section{RESULTADOS E DISCUSSÃO}

\section{Estimativa das propriedades físicas por meio do ultrassom}

Para auxiliar na interpretação dos dados, são apresentados na Tabela 2 os resultados médios do MOED, absorção d'água e inchamento em espessura dos painéis confeccionados com a mistura das diferentes espécies, erva-mate, pinus e eucalipto.

Tabela 2. Valores médios do módulo de elasticidade dinâmico e as propriedades físicas, nos tratamentos avaliados. Table 2. Mean values of dynamic modulus of elasticity and physical properties in the evaluated treatments.

\begin{tabular}{|c|c|c|c|c|c|c|c|c|}
\hline \multirow{2}{*}{ Trat. } & \multicolumn{3}{|c|}{ Partículas (\%) } & \multirow{2}{*}{$\begin{array}{l}\text { MOED } \\
\text { (MPa) }\end{array}$} & \multirow{2}{*}{$\begin{array}{c}\text { AB 2H } \\
(\%) \\
\end{array}$} & \multirow{2}{*}{$\begin{array}{c}\text { AB 24H } \\
(\%)\end{array}$} & \multirow{2}{*}{$\begin{array}{c}\text { IE 2H } \\
(\%) \\
\end{array}$} & \multirow{2}{*}{$\begin{array}{c}\text { IE 24H } \\
(\%)\end{array}$} \\
\hline & Erva-mate & Pinus & Eucalipto & & & & & \\
\hline 1 & 0 & 100 & 0 & 2871,02 & 14,05 & 57,75 & 5,92 & 37,11 \\
\hline 2 & 25 & 75 & 0 & 2763,42 & 18,03 & 64,02 & 7,47 & 29,17 \\
\hline 3 & 50 & 50 & 0 & 2321,52 & 24,41 & 71,60 & 9,66 & 35,65 \\
\hline 4 & 75 & 25 & 0 & 1811,55 & 31,09 & 78,74 & 8,35 & 43,28 \\
\hline 5 & 100 & 0 & 0 & 1657,41 & 48,35 & 85,93 & 12,98 & 39,10 \\
\hline 6 & 0 & 0 & 100 & 2704,65 & 10,56 & 33,13 & 3,23 & 24,93 \\
\hline 7 & 25 & 0 & 75 & 2207,19 & 22,58 & 55,64 & 9,87 & 38,68 \\
\hline 8 & 50 & 0 & 50 & 2261,79 & 33,37 & 72,40 & 14,86 & 39,94 \\
\hline 9 & 75 & 0 & 25 & 2029,83 & 39,26 & 72,16 & 15,00 & 32,94 \\
\hline 10 & 33,3 & 33,3 & 33,3 & 2469,49 & 24,27 & 60,05 & 8,19 & 30,05 \\
\hline 11 & 0 & 50 & 50 & 2893,70 & 8,58 & 38,84 & 3,23 & 25,39 \\
\hline
\end{tabular}

Onde: Trat. = Tratamentos; MOED = Módulo de elasticidade dinâmico; $A B 2 \mathrm{H}=$ Absorção d'água após 2 horas; $A B$ 24H = Absorção d'água após 24 horas; IE $2 \mathrm{H}=$ Inchamento em espessura após 2 horas; IE $24 \mathrm{H}=$ Inchamento em espessura após 24 horas.

Na Tabela 3, observa-se a matriz de correlação entre o MOED e as propriedades físicas dos painéis, para os 11 tratamentos avaliados. Todas as propriedades físicas tiveram correlação significativa a 5\% de probabilidade de erro com o MOED, sendo que, as melhores correlações foram observadas 
para os parâmetros AB 2H (0,9096) e AB 24H (0,7916). Morais (2011), estudando painéis de partículas com diferentes proporções de bambu, pinus e eucalipto, encontrou correlações significativas somente entre MOED e, $\mathrm{AB} 2 \mathrm{H}$ e $\mathrm{AB} 24 \mathrm{H}$.

Tabela 3. Matriz de correlação de Pearson entre o módulo de elasticidade dinâmico e as propriedades físicas dos painéis. Table 3. Pearson correlation matrix between the dynamic modulus of elasticity and the physical properties of the panels.

\begin{tabular}{ccccc}
\hline & AB 2H & AB 24H & IE 2H & IE 24H \\
\hline MOED & $-0,9016^{* \star}$ & $-0,7916^{\star *}$ & $-0,7139^{\star *}$ & $-0,7026^{\star *}$ \\
\hline
\end{tabular}

Onde: ** = Significativo ao nível de $5 \%$ de probabilidade de erro; $M O E D=$ Módulo de elasticidade dinâmico; $A B 2 \mathrm{H}=$ Absorção d'água após 2 horas; $\mathrm{AB} 24 \mathrm{H}=$ Absorção d'água após 24 horas; IE $2 \mathrm{H}=$ Inchamento em espessura após 2 horas; IE $24 \mathrm{H}=$ Inchamento em espessura após 24 horas.

Embora diversas pesquisas venham demonstrando a eficácia de métodos não destrutivos na estimativa das propriedades mecânicas de painéis (MELO; DEL MENEZZI, 2016; SILVA et al. 2015) poucos estudos têm sido desenvolvidos visando a utilização dessa ferramenta na predição de parâmetros relacionados a estabilidade dimensional destes produtos. Apesar de autores como, Miná et al. (2004), explicarem que o ultrassom não deve ser utilizado para a estimação das propriedades físicas, pois os ensaios não destrutivos são baseados nas propriedades mecânicas dos materiais, no presente estudo, com o uso do ultrassom, foi possível estimar de forma significativa os percentuais de absorção d'água e inchamento em espessura dos painéis MDP. Melo e Del Menezzi (2016), analisando painéis estruturais, também obteve significância na estimativa destas propriedades físicas, assim como Morais (2011), com painéis de partículas, obteve significância na estimativa das propriedades de $\mathrm{AB} 2 \mathrm{H}$ e $\mathrm{AB} 24 \mathrm{H}$.

As Figuras 3 e 4 evidenciam os modelos estimados para AB 2H, AB 24H, IE $2 \mathrm{H}$ e IE $24 \mathrm{H}$, em função do MOED. Houve similaridade de comportamento entre as propriedades físicas, já que todas decresceram com o aumento do MOED. Esse comportamento pode ser atribuído ao fato de que em madeiras ou compostos de madeiras, a variável MOED ser diretamente proporcional a sua massa específica (ROSS et al., 1998). Deste modo, conforme observado por Melo e Del Menezzi (2010), uma maior taxa de absorção d'água e inchamento em espessura observada, para amostras com menor MOED, pode ser atribuída ao maior volume de espaços vazios dessas, que possam ser ocupados por água.

Ainda nas Figuras 3 e 4, é possível observar que os maiores coeficientes de determinação da reta ajustada é para $\mathrm{AB} 2 \mathrm{H}$ e $\mathrm{AB} 24 \mathrm{H}$, com $82,75 \%$ e $62,66 \%$, respectivamente. Reforçando assim, os maiores valores de correlação encontrados para estas propriedades, na matriz de correlação de Pearson (Tabela 3).
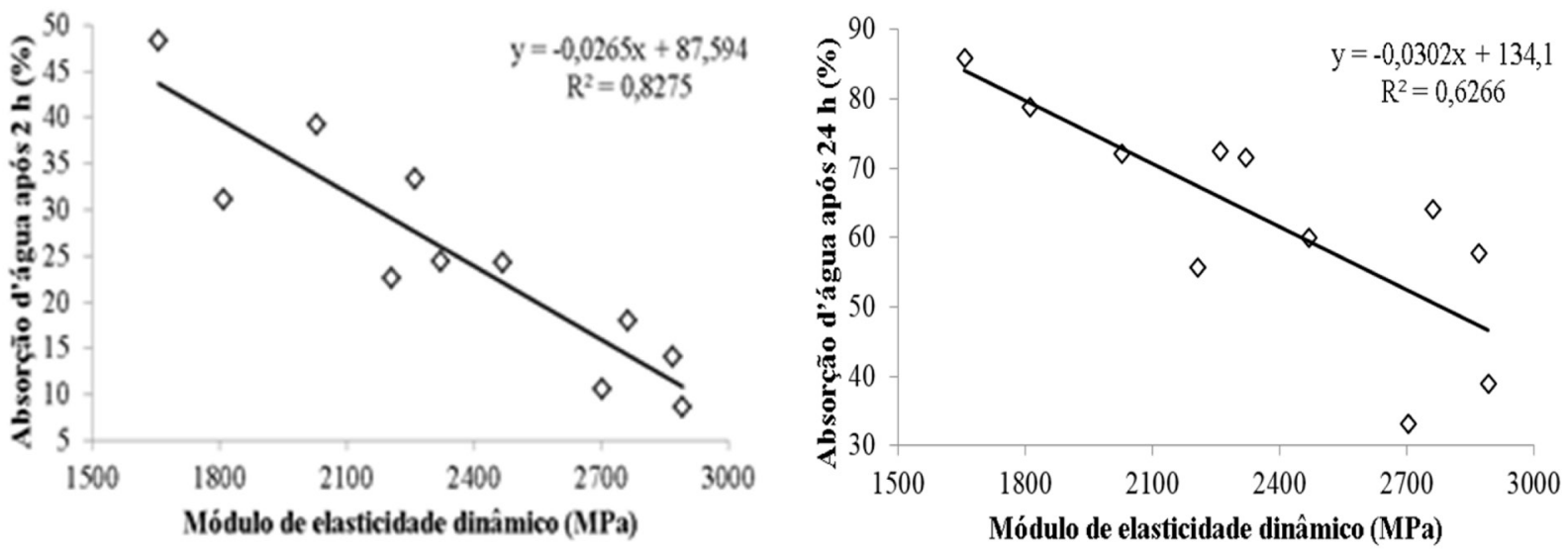

Figura 3. Estimativa da absorção d'água após 2 e 24 horas de imersão, em função do módulo de elasticidade dinâmico obtido por meio do ultrassom.

Figure 3. Estimation of water absorption after 2 and 24 hours of immersion, as a function of the dynamic modulus of elasticity obtained by ultrasound. 

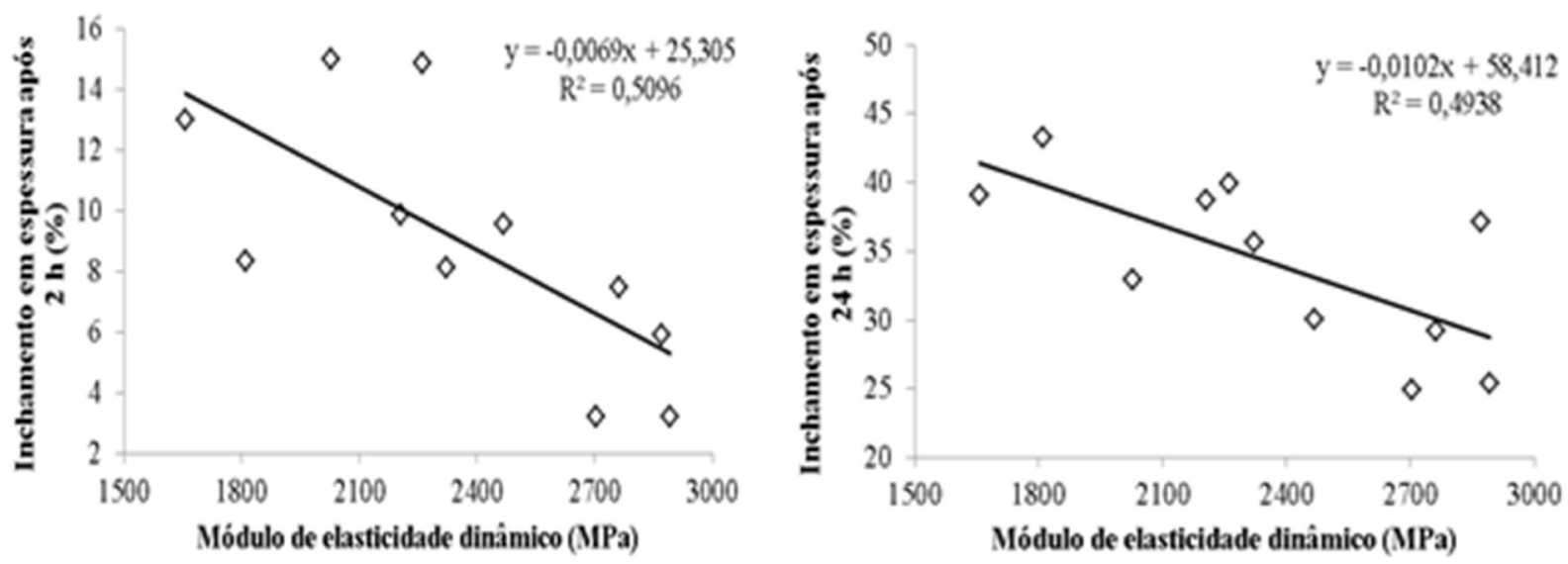

Figura 4. Estimativa do inchamento em espessura após 2 e 24 horas de imersão, em função do módulo de elasticidade dinâmico obtido por meio do ultrassom.

Figure 4. Estimation of swelling in thickness after 2 and 24 hours of immersion, as a function of the dynamic modulus of elasticity obtained by ultrasound.

\section{Estimativa das propriedades mecânicas por meio do ultrassom}

A Tabela 4 apresenta os resultados médios do MOED e, MOE e MOR de flexão estática dos painéis confeccionados com a mistura das diferentes espécies, erva-mate, pinus e eucalipto.

Tabela 4. Valores médios estimados do módulo de elasticidade dinâmico e as propriedades mecânicas de flexão estática, nos tratamentos avaliados.

Table 4. Mean estimation values of the modulus of dynamic elasticity and the mechanical properties of static bending in the evaluated treatments.

\begin{tabular}{lcccccc}
\hline \multirow{2}{*}{ Trat. } & Erva-mate & Pinus & Eucalipto & $\begin{array}{c}\text { MOED } \\
\text { (MPa) }\end{array}$ & $\begin{array}{c}\text { MOE } \\
\text { (MPa) }\end{array}$ & $\begin{array}{c}\text { MOR } \\
\text { (MPa) }\end{array}$ \\
\hline 1 & 0 & 100 & 0 & 2871,02 & 2208,82 & 13,74 \\
2 & 25 & 75 & 0 & 2763,42 & 2470,98 & 13,76 \\
3 & 50 & 50 & 0 & 2321,52 & 2214,32 & 10,36 \\
4 & 75 & 25 & 0 & 1811,55 & 1655,20 & 7,74 \\
5 & 100 & 0 & 0 & 1657,41 & 1181,45 & 4,84 \\
6 & 0 & 0 & 100 & 2704,65 & 2065,37 & 13,67 \\
7 & 25 & 0 & 75 & 2207,19 & 1916,81 & 9,76 \\
8 & 50 & 0 & 50 & 2261,79 & 1749,80 & 9,30 \\
9 & 75 & 0 & 25 & 2029,83 & 1729,96 & 8,86 \\
10 & 33,33 & 33,33 & 33,33 & 2469,49 & 2101,46 & 11,43 \\
11 & 0 & 50 & 50 & 2893,70 & 2435,49 & 14,50 \\
\hline
\end{tabular}

Em que: Trat. = Tratamentos; $\mathrm{MOED}=$ Módulo de elasticidade dinâmico; $\mathrm{MOE}=$ Módulo de elasticidade estático; MOR = Módulo de ruptura.

Por meio da Tabela 5, é possível analisar a matriz de correlação entre MOED e, MOE e MOR dos painéis, para os 11 tratamentos avaliados. Houve correlação significativa a 5\% de probabilidade de erro entre o MOED e as propriedades de flexão, MOE e MOR, sendo a melhor correlação para MOR $(0,9832)$.

Tabela 5. Matriz de correlação de Pearson entre o módulo de elasticidade dinâmico e as propriedades mecânicas de flexão estática dos painéis.

Table 5. Pearson correlation matrix between the dynamic modulus of elasticity and the mechanical properties of static flexion of the panels.

\begin{tabular}{ccc} 
& MOE & MOR \\
\hline MOED & $0,9037^{* *}$ & $0,9832^{* *}$
\end{tabular}

Em que: ${ }^{*} *$ = significativo em nível de $5 \%$ de probabilidade de erro; MOED = Módulo de elasticidade dinâmico; MOE = Módulo de elasticidade estático; MOR = Módulo de ruptura.

Jayne (1959), propôs a hipótese fundamental para o uso do ultrassom em madeiras. O autor afirma que as propriedades anatômicas e químicas da madeira, que servem para armazenar e dissipar a energia do ultrassom são controladas pelos mesmos mecanismos que determinam o seu comportamento mecânico em condições estáticas. Desse modo, ao comparar-se o comportamento 
do MOED com o MOR e com MOE, nota-se comportamentos similares, uma vez que o aumento do MOR e MOE aumenta o MOED (Figura 6).

Jayne (1959) e Stangerlin et al. (2011) observaram, experimentalmente, nos respectivos estudos, relações entre os ensaios não destrutivos e os mecânicos. Porém, Lapo e Beraldo (2008) não encontraram relações entre o MOED e o MOR utilizando o método de propagação direto, e recomendam o uso da propagação da onda ultrassônica ao longo da espessura do painel, varrendo-se uma grande quantidade de pontos. Todavia, no presente estudo foi observada a correlação entre o MOED e o MOR, não sendo necessária a amostragem em vários pontos dos corpos de prova.

De acordo com a Tabela 5 e a Figura 5, o MOR apresentou melhor correlação com o MOED, fato também observado por Morais (2011), estudando painéis de partículas com diferentes proporções de bambu, pinus e eucalipto. O mesmo não foi observado por Halabe et al. (1995), ao correlacionar o MOE e MOR com as constantes elásticas dinâmicas obtidas mediantes ensaios de propagação de ondas ultrassonoras em madeira verde de diferentes espécies. Conforme Miná et al. (2004) e Oliveira et al. (2003), este comportamento se deve ao fato de que as medições dinâmicas são baseadas nas propriedades mecânicas apenas no limite elástico. O MOR acontece em maior tensão e depois do limite elástico, resultando, consequentemente, em predições menos eficazes por meio dos ensaios não destrutivos. O MOED obtido pelo método ultrassonoro foi superior ao MOE verificado pelo ensaio destrutivo. Este resultado está de acordo com o obtido por Morais (2011), ao estudarem painéis de partículas.

Corroborando, Oliveira et al. (2003) explicam que na vibração de uma espécie de madeira, a força elástica restaurada é proporcional ao deslocamento, e a força dissipativa é proporcional à velocidade. Portanto, quando a força é aplicada com curta duração, o material se comporta como um sólido elástico, enquanto que em uma longa duração o comportamento é igual ao de um líquido viscoso. Tal comportamento é mais proeminente no ensaio de flexão estática, o qual apresenta longa duração se comparado ao ensaio de ultrassom. Em consequência desse comportamento, o MOED obtido em ensaio de ultrassom é, em geral, maior que o MOE à flexão estática.
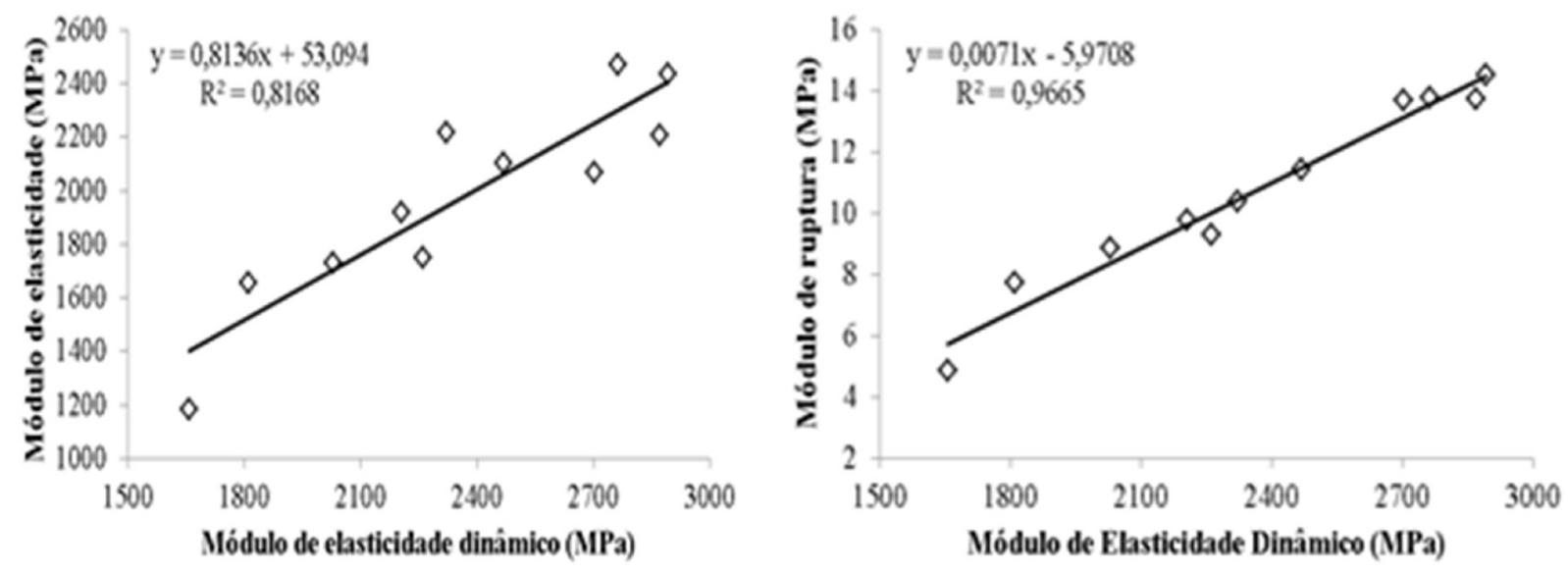

Figura 5. Estimativa do módulo de elasticidade estático e do módulo de ruptura, em função do módulo de elasticidade dinâmico obtido por meio do ultrassom.

Figure 5. Estimation of the static modulus of elasticity and the modulus of rupture, as a function of the dynamic modulus of elasticity obtained by ultrasound.

A Tabela 6 apresenta os resultados médios do MOED, AP Face, AP Topo, TP e RS dos painéis confeccionados com a mistura das diferentes espécies, erva-mate, pinus e eucalipto.

A matriz de correlação entre MOED e, AP Face, AP Topo, TP e RS dos painéis, para os 11 tratamentos avaliados, está presente na Tabela 7. Para todas estas propriedades mecânicas avaliadas, não houve correlação significativa a 5\% de probabilidade de erro. Comportamento inverso foi observado por Morais (2011), ao analisar estas mesmas características em painéis de partículas. 
Tabela 6. Valores médios estimados do módulo de elasticidade dinâmico e as propriedades mecânicas, nos tratamentos avaliados.

Table 6. Mean estimation values of the modulus of dynamic elasticity and the mechanical properties in the evaluated treatments.

\begin{tabular}{|c|c|c|c|c|c|c|}
\hline \multirow{2}{*}{ Trat. } & \multicolumn{3}{|c|}{ Partículas (\%) } & \multirow{2}{*}{$\begin{array}{c}\text { MOED } \\
(\mathrm{MPa})\end{array}$} & \multirow{2}{*}{$\begin{array}{c}\text { MOE } \\
\text { (MPa) }\end{array}$} & \multirow{2}{*}{$\begin{array}{c}\text { MOR } \\
\text { (MPa) }\end{array}$} \\
\hline & Erva-mate & Pinus & Eucalipto & & & \\
\hline 1 & 0 & 100 & 0 & 2871,02 & 2208,82 & 13,74 \\
\hline 2 & 25 & 75 & 0 & 2763,42 & 2470,98 & 13,76 \\
\hline 3 & 50 & 50 & 0 & 2321,52 & 2214,32 & 10,36 \\
\hline 4 & 75 & 25 & 0 & 1811,55 & 1655,20 & 7,74 \\
\hline 5 & 100 & 0 & 0 & 1657,41 & 1181,45 & 4,84 \\
\hline 6 & 0 & 0 & 100 & 2704,65 & 2065,37 & 13,67 \\
\hline 7 & 25 & 0 & 75 & 2207,19 & 1916,81 & 9,76 \\
\hline 8 & 50 & 0 & 50 & 2261,79 & 1749,80 & 9,30 \\
\hline 9 & 75 & 0 & 25 & 2029,83 & 1729,96 & 8,86 \\
\hline 10 & 33,33 & 33,33 & 33,33 & 2469,49 & 2101,46 & 11,43 \\
\hline 11 & 0 & 50 & 50 & 2893,70 & 2435,49 & 14,50 \\
\hline
\end{tabular}

Em que: Trat. = Tratamentos; MOED = Módulo de elasticidade dinâmico; AP Face = arrancamento de parafusos na face; $\mathrm{AP}$ Topo = arrancamento de parafusos no topo; TP = tração perpendicular à superfície do painel; RS = resistência superficial.

Tabela 7. Matriz de correlação de Pearson entre o módulo de elasticidade dinâmico e as propriedades mecânicas dos painéis.

Table 7. Pearson correlation matrix between the dynamic modulus of elasticity and the mechanical properties of the panels.

\begin{tabular}{lcccc}
\hline & AP FACE & AP TOPO & TP & RS \\
\hline MOED & 0,3294 & 0,5656 & $-0,5796$ & 0,0683 \\
\hline
\end{tabular}

Em que: ** = significativo em nível de $5 \%$ de probabilidade de erro; MOED = Módulo de elasticidade dinâmico; AP Face = arrancamento de parafusos na face; AP Topo = arrancamento de parafusos no topo; TP = tração perpendicular à superfície do painel; RS $=$ resistência superficial.

\section{Avaliação colorimétrica}

A Tabela 8 apresenta os valores médios dos parâmetros colorimétricos das superfícies dos 11 tratamentos de painéis MDP, produzidos com diferentes proporções de partículas madeireiras de erva-mate, pinus e eucalipto. Observa-se que todos os parâmetros colorimétricos apresentaram efeito significativo a $5 \%$ de probabilidade de erro, entre os tratamentos avaliados. Todos os painéis apresentaram valores de $\mathrm{a}^{*} \mathrm{e} \mathrm{b}^{*}$ positivos, o que classifica as amostras no primeiro quadrante de cores no Sistema CIELAB 1976, padrão de comportamento esperado para a coloração de madeiras.

Tabela 8. Valores médios dos parâmetros colorimétricos pelo Teste de Scott - Knott, nos tratamentos avaliados.

Table 8. Mean values of the colorimetric parameters by the Scott-Knott test in the evaluated treatments.

\begin{tabular}{lccc|ccccc}
\hline \multirow{2}{*}{ Trat. } & \multicolumn{3}{c|}{ Partículas (\%) } & \multicolumn{5}{c}{ Parâmetros Colorimétricos } \\
\cline { 2 - 8 } & Erva-mate & Pinus & Eucalipto & $\mathbf{L}^{*}$ & $\mathbf{a}^{*}$ & $\mathbf{b}^{*}$ & $\mathbf{C}$ & $\mathbf{h}^{*}$ \\
\hline 1 & 0 & 100 & 0 & $75,99 \mathrm{a}$ & $4,08 \mathrm{~g}$ & $29,69 \mathrm{a}$ & $29,98 \mathrm{a}$ & $82,17 \mathrm{a}$ \\
2 & 25 & 75 & 0 & $73,64 \mathrm{~b}$ & $4,07 \mathrm{~g}$ & $26,09 \mathrm{~b}$ & $26,41 \mathrm{~b}$ & $81,15 \mathrm{~b}$ \\
3 & 50 & 50 & 0 & $70,54 \mathrm{c}$ & $4,06 \mathrm{~g}$ & $25,57 \mathrm{c}$ & $25,91 \mathrm{~b}$ & $81,01 \mathrm{~b}$ \\
4 & 75 & 25 & 0 & $69,13 \mathrm{~d}$ & $4,44 \mathrm{~g}$ & $26,14 \mathrm{~b}$ & $26,52 \mathrm{~b}$ & $80,38 \mathrm{~b}$ \\
5 & 100 & 0 & 0 & $67,33 \mathrm{e}$ & $4,04 \mathrm{~g}$ & $25,23 \mathrm{c}$ & $25,55 \mathrm{c}$ & $80,91 \mathrm{~b}$ \\
6 & 0 & 0 & 100 & $68,10 \mathrm{e}$ & $8,76 \mathrm{a}$ & $21,13 \mathrm{~g}$ & $22,88 \mathrm{~g}$ & $67,48 \mathrm{f}$ \\
7 & 25 & 0 & 75 & $66,71 \mathrm{f}$ & $7,92 \mathrm{~b}$ & $22,16 \mathrm{f}$ & $23,53 \mathrm{f}$ & $70,32 \mathrm{e}$ \\
8 & 50 & 0 & 50 & $68,06 \mathrm{e}$ & $6,50 \mathrm{~d}$ & $22,07 \mathrm{f}$ & $23,02 \mathrm{~g}$ & $73,58 \mathrm{~d}$ \\
9 & 75 & 0 & 25 & $65,64 \mathrm{f}$ & $5,89 \mathrm{e}$ & $24,10 \mathrm{~d}$ & $24,81 \mathrm{~d}$ & $76,26 \mathrm{c}$ \\
10 & 33,33 & 33,33 & 33,33 & $69,96 \mathrm{~d}$ & $5,41 \mathrm{f}$ & $23,63 \mathrm{e}$ & $24,25 \mathrm{e}$ & $77,11 \mathrm{c}$ \\
11 & 0 & 50 & 50 & $70,84 \mathrm{c}$ & $7,15 \mathrm{c}$ & $24,38 \mathrm{~d}$ & $25,41 \mathrm{c}$ & $73,64 \mathrm{~d}$ \\
\hline
\end{tabular}

Em que: Trat. = Tratamentos; $\mathrm{L}^{*}=$ Grau de claridade; $\mathrm{a}^{*}=$ Matizes do eixo vermelho $(+)$ e verde $(-) ; \mathrm{b}^{*}=$ Matizes do eixo amarelo $(+)$ e azul (-); C = Saturação da cor; $\mathrm{h}^{*}=$ Ângulo de tinta.

Ao analisar os valores médios dos parâmetros colorimétricos obtidos no estudo, observa-se que o grau de claridade ( $\mathrm{L}^{*}$ ) teve os maiores valores nos tratamentos 1, 2, 3 e 11, diminuindo de maneira significativa a claridade, em função da menor porcentagem de pinus na composição do painel. Sendo assim, o maior grau de claridade foi para o tratamento 1, que possui $100 \%$ de pinus na sua composição. Os menores valores de $L^{*}$ ficaram para os tratamentos 7 e 9, em função da maior 
quantidade de partículas de eucalipto e erva-mate. Sabe-se que o eucalipto é uma madeira de menor claridade que o pinus, entretanto esperava-se que a erva-mate também colaborasse para valores mais altos de $\mathrm{L}^{*}$. Esse comportamento distinto ocorreu devido ao aparecimento de leves queimaduras na superfície dos painéis MDP com partículas de erva-mate na sua composição. O grau de queimadura foi maior quanto maior a proporção da referida espécie. Porém, uma vez que todos os valores de $\mathrm{L}^{*}$ apresentaram valores acima de 56, independentemente de sua composição, os painéis podem ser classificados como cores claras (LIMA et al., 2013).

Os painéis feitos com proporções de partículas de eucalipto apresentaram uma cor rosada, sendo maior está tonalidade quanto maior a quantidade desta espécie na composição do painel. Isso é resultado dos valores mais altos do pigmento vermelho $\left(\mathrm{a}^{*}\right)$, que possui pico máximo no tratamento 6, com $100 \%$ de eucalipto, e diminui significativamente segundo o teste de média de Scott - Knott, conforme reduz-se a proporção da referida espécie.

Para os painéis MDP feitos exclusivamente de partículas de pinus (tratamento 1), o parâmetro determinante foi o $\mathrm{b}^{*}$, em detrimento do baixo valor de $\mathrm{a}^{*}$, que leva o painel a ser classificado como de cor amarela. Os tratamentos que receberam diferentes combinações de erva-mate e pinus também tiveram valores altos para $\mathrm{o} \mathrm{b*}$, entretanto conforme aumenta-se a quantidade de erva-mate e diminui-se de pinus, este parâmetro reduz significativamente. O grau de queimadura na superfície destes painéis com diferentes proporções de erva-mate, embora baixo, também pode ter influenciado na mensuração deste parâmetro. Castro et al. (2014), estudando aglomerados em diferentes combinações de Sequoia sempervirens e Pinus taeda, encontrou valores semelhantes para b*, nos painéis com maior quantidade de pinus, apresentando maior tendência a coloração amarela. Corroborando com o expoto, Gonçalez et al. (2001), a coordenada a*, quando positiva e crescente, significa presença da coloração avermelhada mais pronunciada e quando a coordenada $b^{*}$ aumenta, significa a presença da cor amarela.

Já quanto ao ângulo de tinta $\left(\mathrm{h}^{*}\right)$ e a saturação da cor $(\mathrm{C})$, os mesmos seguem padrão semelhante de comportamento às coordenadas $\mathrm{a}^{*} \mathrm{e} \mathrm{b}^{*}$, pois são diretamente influenciadas por estas variáveis. $\mathrm{O} \mathrm{h}^{*}$, neste estudo, ajudou a definir o padrão de cor dos MDPs de pinus, tendo em vista que seu alto valor faz os painéis se aproximar do eixo $\mathrm{b}^{*}$. O parâmetro $\mathrm{C}$ também é mais evidente em tratamentos com maior proporção de partículas de pinus, do que daqueles de partículas de erva-mate e eucalipto. Os valores médios de todos os parâmetros dos MDPs produzidos com maior quantidade de partículas de pinus, mostraram-se semelhantes aos apresentados por Castro et al. (2013), para partículas de Pinus taeda. Essa semelhança indica que o painel mantém as características colorimétricas da madeira utilizada em sua formação, com pouca influência do adesivo ureia-formaldeído.

Segundo Castro et al. (2014), os tratamentos feitos a partir de misturas de espécies apresentaram parâmetros intermediários entre os painéis produzidos com partículas de apenas uma espécie. O aumento da proporção de partículas de pinus tende a diminuir a influência do eixo vermelho ( $\left.\mathrm{a}^{*}\right)$ e aumentar a do eixo amarelo $\left(b^{*}\right)$, fato confirmado com o aumento do angulo de tinta $\left(h^{*}\right)$.

Na Figura 6 é possível verificar, claramente, a influência das misturas das três espécies estudas, bem como a coloração em MDPs puros para cada espécie. Segundo Mori et al. (2005), o padrão de coloração de uma madeira pode variar em tonalidades que vão desde o bege claro até o marrom escuro, quase preto; dentro destas variações, podem ser encontradas madeiras amareladas, avermelhadas, arroxeadas e alaranjadas.

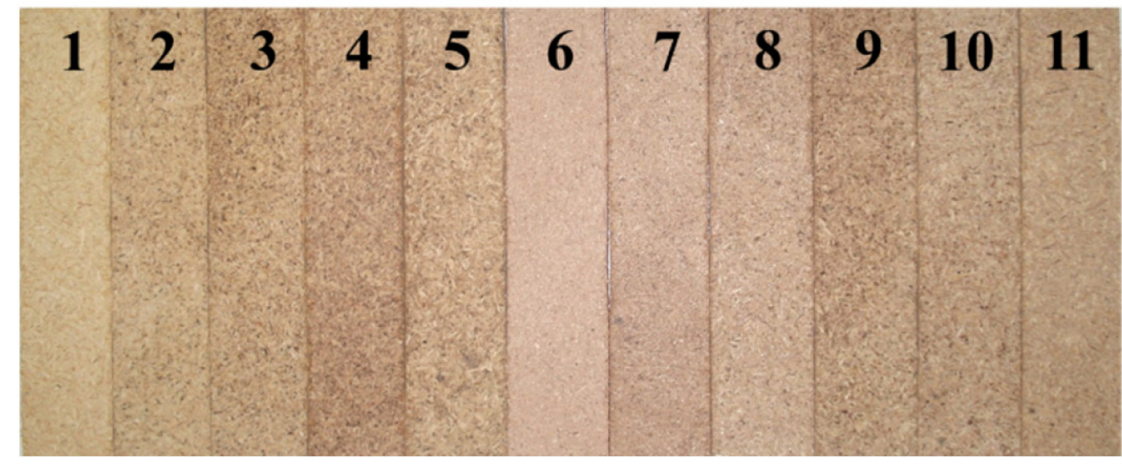

Figura 6. Imagem da superfície dos painéis MDP, para os 11 tratamentos avaliados.

Figure 6. Surface image of MDP panels for the 11 treatments evaluated. 


\section{CONCLUSÕES}

O método de avaliação não destrutiva utilizando ultrassom pode ser utilizado na predição das propriedades físicas dos painéis MDP, produzidos com partículas de madeira de erva-mate, pinus e eucalipto. Entretanto, para as propriedades mecânicas exceções foram observadas para o AP Face, AP Topo, TP e RS, onde o uso do ultrassom não possibilitaram ajustes de modelos significativos. Para as propriedades mecânicas de flexão estática, MOE e MOR, o ultrassom possibilitou as melhores estimativas do presente estudo. As correlações significativas do MOED, em ordem de significância, ocorreram com o MOR, MOE, AB 2H, AB 24H, IE 2H e IE 24H.

Os MDPs tendem a manter a aparência colorimétrica da espécie das partículas utilizadas no processo. Painéis de eucalipto apresentaram uma coloração rosada, enquanto que os produzidos com partículas de pinus e erva-mate apresentaram cor amarelada, sendo esta coloração mais pronunciada em painéis com maior proporção de partículas de pinus. Dessa forma, a indústria de MDPs pode apresentar produtos com novos padrões de cores a partir de uma produção com mistura de partículas de diferentes espécies.

\section{REFERÊNCIAS BIBLIOGRÁFICAS}

CASTRO, V. G.; BRAZ, R. L.; BELLON, K.; SANCHES, F.; IWAKIRI, S.; NISGOSKI, S. Avaliação colorimétrica de aglomerados produzidos com partículas de madeira de Sequoia sempervirens e Pinus taeda submetidos ao intemperismo natural. Floresta e Ambiente, Seropédica, v. 21, n. 4, p. 475-483, 2014. DOI: http://dx.doi. org/10.1590/2179-8087.021713.

CASTRO, V. G.; KLOCK, U.; IWAKIRI, S.; BOLZON, G. I. Avaliação colorimétrica de partículas de Pinus taeda submetidas a diferentes métodos de acetilação. Scientia Forestalis, Piracicaba, v. 41, n. 98, p. 265-270, 2013.

GONÇALEZ, J. C.; JANIN, G.; SANTORO, A. C. S.; COSTA, A.F.; VALLE, A. T. Colorimetria quantitativa: uma técnica objetiva de determinar a cor da madeira. Revista Brasil Florestal, n. 72. p. 47 - 48. 2001.

HALABE, U. B.; BIDIGALU, G. M.; GANGARAO, H. V. S.; ROSS, R. J. Nondestructive evaluation of green wood using stress wave and transverse vibration techniques. Materials evaluation, v. 55, n. 9, p. 1013-1018, 1995.

HAN, G.; WU, Q.; WANG, X. Stress-wave velocity of wood based panels: effect of moisture, product type, and material direction. Forest Products Journal, v. 56, n. 1, p. 28-33, 2006.

JAYNE, B. A. Vibrational properties of wood as indices of quality. Forest Products Journal. v. 9, n. 11, p. 413-416, 1959.

LAPO, L. E. R.; BERALDO, A. L. Avaliação de características de chapas de partículas de bambu e adesivo poliuretana à base de óleo de mamona. In: ENCONTRO BRASILEIRO EM MADEIRA E ESTRUTURAS DE MADEIRA, 11., 2008, Londrina. Anais eletrônicos... Londrina: UEL, 2008. Disponível em: < http://www. ebramem.expertu.com.br >.Acesso em: 23 Out. 2016.

LIMA, C.L.; GONÇALEZ, J.C.; COSTA, T.R.V.; PEREIRA, R.S.; LIMA, J.B.M.; LIMA, M.A.S. Comportamento da cor de lâminas de madeira de Pau-marfim (Balfourodendron riedelianum) tratada com produtos de acabamento. Revista Árvore, Curitiba, v. 37, n. 2, p. 377-384, 2013. DOI: http://dx.doi.org/10.1590/S010067622013000200020 .

MELO, R. R.; DEL MENEZZI, C. H.S. Estimativas das propriedades físico-mecânicas de compostos LVL confeccionados com paricá por meio de ultrassom. Ciência Florestal, Santa Maria, v. 26, n. 1, p. 263-272, 2016. DOI: http://dx.doi.org/10.1590/S0100-67622014000600020

MELO, R. R.; DEL MENEZZI, C. H. S. Influência da massa específica nas propriedades físico-mecânicas de painéis aglomerados. Revista Silva Lusitana, Lisboa, v. 18, n. 1, p. 59-73, 2010.

Sci. For., Piracicaba, v. 46, n. 119, p. 507-518, set. 2018 DOI: dx.doi.org/10.18671/scifor.v46n119.17 
Souza et al. - Propriedades não destrutivas do MDP produzido com partículas de madeiras e de Ilex paraguariensis

MINÁ, A. J. S.; OLIVEIRA, F. G. R.; JUNIOR, C. C.; DIAS, A. A.; SALES, A. Avaliação não destrutiva de postes de madeira por meio de ultrassom. Revista Scientia Forestalis, Piracicaba, v. 1, n. 65, p. 188-189, 2004.

MORAIS, W. W. C. Propriedades físico-mecânicas de chapas aglomeradas produzidas com bambu, pinus e eucalipto. 2011. 132 p. Dissertação (Mestrado em Engenharia Florestal) - Universidade Federal de Santa Maria, Santa Maria, 2011.

OLIVEIRA, F. G. R.; CANDIAN, M.; LUCCHETTE, F. F.; JUNIOR, C. C.; SALES, A. Avaliação de propriedades mecânicas de madeira por meio de ultra-som. In: PAN AMERICAN CONFERENCE FOR NONDESTRUCTIVE TESTING, 3., 2003, Rio de Janeiro. Anais... Rio de Janeiro: Pan American Conference for Nondestructive Testing, 2003. 5 p.

PELLERIN, R. F.; ROSS, J. R. Nondestructive evaluation of wood. Madison: FPS, 2002. 210 p.

ROSS, R.J.; ERICKSON, J. R.; BRASHAW, B. K.; WANG, X.; VERHEY, S. A.; FORSMAN, J. W.; PILON, C. L. Yield and ultrasonic modulus of elasticity of red maple veneer. Forest Products Journal, v. 54, n.12, p.220-225, 2004.

ROSS, R. J.; BRASHAW, B. K.; PELLERIN, R. F. Nondestructive evaluation of wood. Forest Products Journal, v. 48, n. 1, p. $14-19,1998$.

SILVA, E. S.; STANGERLIN, D. M.; GATTO, D. A.; CALEGARI, L.; PARIZ, E. Colorimetria da madeira de oito espécies nativas do estado do Rio Grande do Sul, Brasil. Ciência da Madeira, Pelotas, n. 6, v. 1, p. 31-37, 2015. DOI: $10.12953 / 2177-6830 /$ rcm.v6n1p31-37.

STANGERLIN, R. R.; GARLET, A.; GATTO, D. A. Durabilidade natural de painéis aglomerados confeccionados com Eucalyptus grandis e Bambusa vulgaris em ensaio de apodrecimento acelerado. Ciência Rural, Santa Maria, v. 41, n.8, 2011. DOI: http://dx.doi.org/10.1590/S0103-84782011000800012.

Recebido em 20/09/2017

Aceito em 15/03/2018 